\title{
Single-port robot-assisted prosthetic breast reconstruction with the da Vinci SP Surgical System: first clinical report
}

\author{
Oh Young Joo ${ }^{1}$, Seung Yong Song ${ }^{1}$, Hyung Seok Park ${ }^{2}$, Tai Suk Roh ${ }^{1}$ \\ Departments of ${ }^{1}$ Plastic and Reconstructive Surgery and ${ }^{2}$ Surgery, Yonsei University College of Medicine, Seoul, Korea
}

\begin{abstract}
Robot-assisted nipple-sparing mastectomy with immediate reconstruction is currently performed in an attempt to seek smaller and indistinct incisions. Robotic surgery system has been evolving under the concept of minimal invasive technique which is a recent trend in surgery. One of the latest version is the da Vinci SP Surgical System (Intuitive Surgical). In this report, we will share our experiences. Two patients underwent robot-assisted nipple-sparing mastectomy, each followed by immediate robot-assisted expander insertion and prepectoral direct-to-implant breast reconstruction, respectively. There was no open conversion or major postoperative complication. One patient experienced mild infection, which was resolved by intravenous antibiotic treatment. Simple docking process, multi-joint instruments, and thirdarm functionality are among the new surgical system's advantages. The present cases suggest that robot-assisted nipple-sparing mastectomy with immediate reconstruction using the da Vinci SP Surgical System is feasible and safe. The promising features and potential application of da Vinci SP in breast reconstruction need further study.
\end{abstract}

Keywords Da Vinci SP / Robotic surgery / Mammaplasty
Correspondence: Seung Yong Song Department of Plastic and Reconstructive Surgery, Yonsei University College of Medicine, 50-1 Yonsei-ro, Seodaemun-gu, Seoul 03722, Korea

Tel: +82-2-2228-2214

Fax: +82-2-393-6947

E-mail: pssysong@yuhs.ac

Received: July 29, 2020 • Revised: December 3, 2020 • Accepted: December 10, 2020

pISSN: 2234-6163 • elSSN: 2234-6171 • https://doi.org/10.5999/aps.2020.01557• Arch Plast Surg 2021;48:194-198

\section{INTRODUCTION}

With its three-dimensional imaging system and flexible robotic arms and instruments, robotic surgery is being carried out in many different fields of surgeries $[1,2]$. Through a very small axillary wound, the da Vinci surgical platform is introduced to perform robot-assisted nipple-sparing mastectomy (R-NSM) [3], with immediate breast reconstruction (IBR). This surgical technique is reported to have shown promising surgical and cosmetic outcomes $[4,5]$.

The recently released da Vinci SP Surgical System (Intuitive Surgical, Sunnyvale, CA, USA) is a true single-port platform with all the instruments entering into one single port [6]. The newly improved single-port design offers three fully articulating 6-mm instruments, as opposed to rigid instrument arms of current robotic systems. Instead of each instrument approaching the surgical site from a different trajectory, the da Vinci SP instruments all pass through a single 25 -mm cannula port and individually articulate within the surgical field [7].

We present two patients who underwent R-NSM and IBR with satisfactory results. To our knowledge, these cases are the first breast reconstruction cases using the da Vinci SP Surgical System. 


\section{CASES}

Two patients were referred to our breast cancer clinic for breast cancer. Based on the patients' breast size and their will to reduce surgical scars, we proposed R-NSM and IBR.

Nipple-sparing mastectomy along with sentinel lymph node biopsy were performed by a single oncologic surgeon (HSP) using the da Vinci SP Surgical System through a longitudinal skin incision in the mid-axillary line. The lengths of the incisions were $4.7 \mathrm{~cm}$ (right side), $3.7 \mathrm{~cm}$ (left side) for one patient and $2.5 \mathrm{~cm}$ for the other patient. The lengths of the incisions were not homogenous because these cases were initial trials. After some experience, the incision length is getting shorter and shorter. Additional incisions (resulting length was $5 \mathrm{~cm}$ for both patients) had to be made by the plastic surgeon (SYS) for the insertion of breast implant or tissue expander. The breast tissue was detached and removed through the same axillary incision. After mastectomy, the EndoWrist instruments (Intuitive Surgical Inc., Sunnyvale, CA, USA) were sterilized for re-use during the reconstruction procedure. The resulting scars were not able to be seen in frontal view and was hidden by the patient's arm in lateral view (Fig. 1).

\section{Case 1}

The first patient desired one-staged breast reconstruction, thus we planned for prepectoral direct-to-implant breast reconstruction. A $16 \times 16-\mathrm{cm}$ sized sheet of acellular dermal matrix (ADM; MegaDerm, L\&C BIO, Seongnam, Korea) was sewn to provide total coverage of the prosthesis (Fig. 2). After preparation of a prepectoral pocket which was measured and designed based on the diameter of the desired implant, two 200-mL negative drainage systems were inserted, then irrigation with saline containing

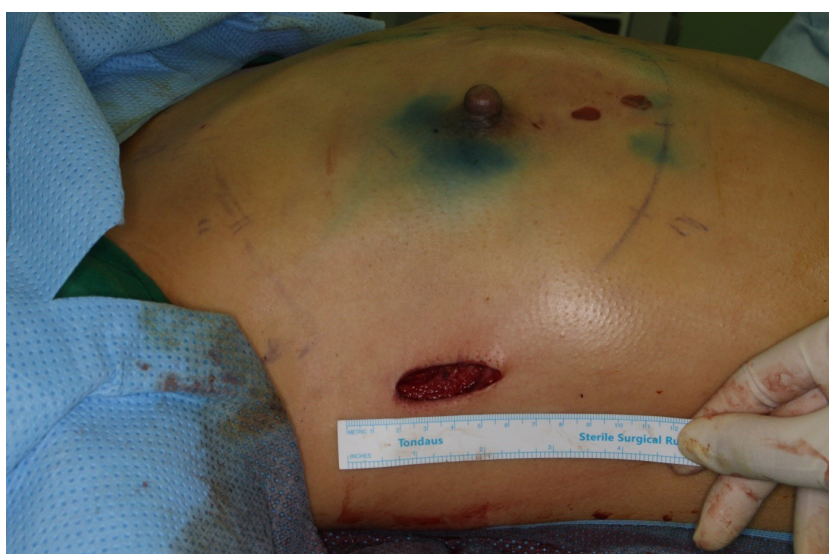

Fig. 1. Incision on axilla. Four centimeters incision on axilla is noted. Robot-assisted nipple-sparing mastectomy and sentinel lymph node biopsy was performed by our institute's oncologic surgeon. antibiotics was done. Then, the ADM-covered implant was inserted to the prepectoral pocket with the assist of a funnel (Art funnel, Art Meditech, Seoul, Korea) (Fig. 3). After insertion, $\mathrm{ADM}$ was sutured manually to the upper and lateral side of pectoralis major muscle. Robotic arms were not used in this case.

During the procedure we have checked the blood circulation in the mastectomy flap as well as the position of inframammary fold (IMF). In this case, the IMF line was well preserved during mastectomy. The burn wounds seen in Fig. 1 were caused unintentionally during mastectomy and were carefully managed by our plastic surgery team. We carefully applied brassiere to maintain proper position of implant and to the extent that it does not interfere with perfusion of the mastectomy flap.

\section{Case 2}

The second patient wanted augmented breasts and thus we recommended two-staged breast reconstruction with tissue expander insertion. Since we think that implant insertions into the same plane for both breasts would be more comfortable to the patient, subpectoral tissue expander insertion was decided. Pos-
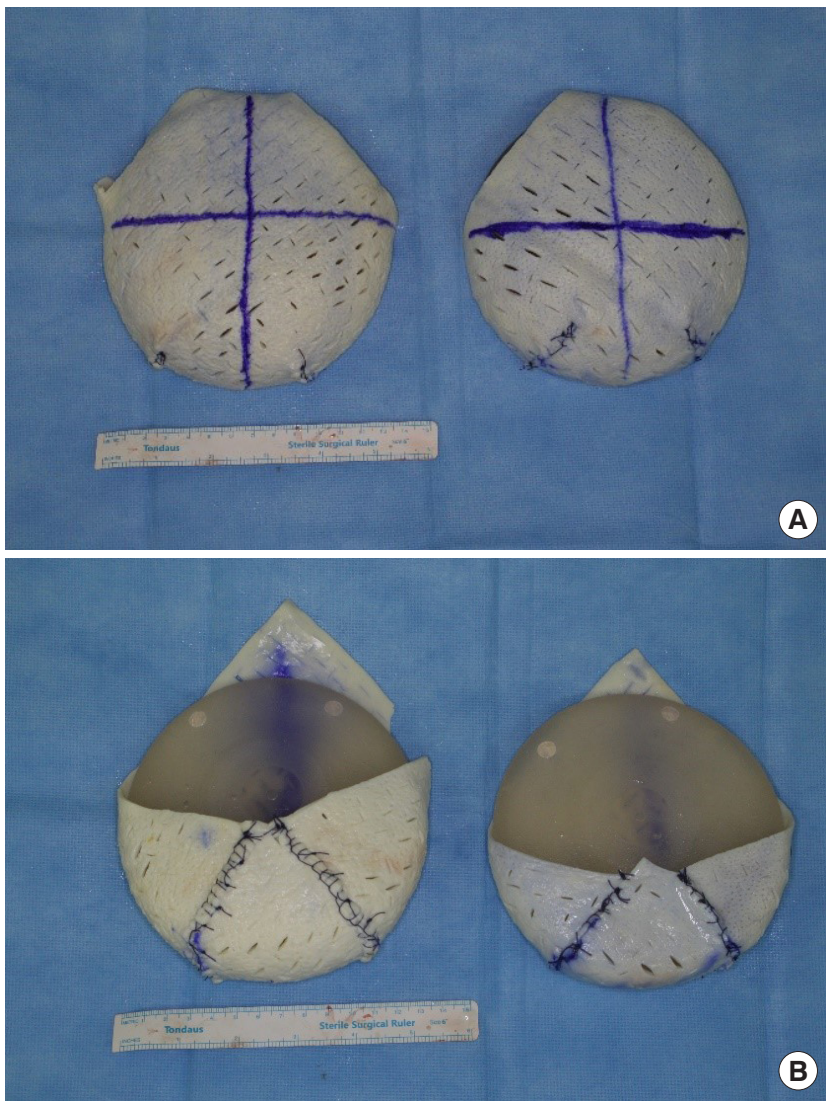

Fig. 2. ADM coverage for prepectoral DTI reconstruction. (A) Anteroposterior view. (B) Posteroanterior view. One sheet of ADM was designed and sewn to fully cover the implant. ADM, acellular dermal matrix; DTI, direct-to-implant. 


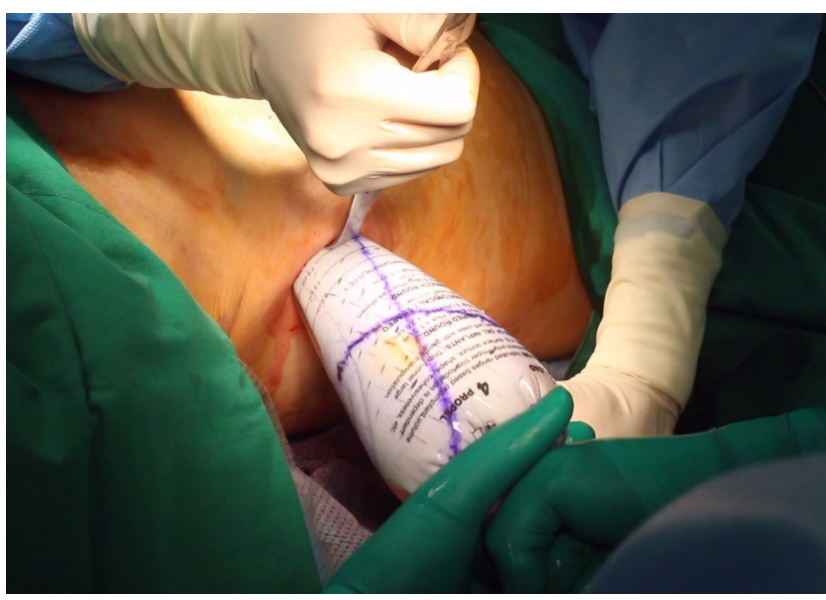

Fig. 3. ADM-wrapped implant insertion through funnel kit. ADM, acellular dermal matrix.

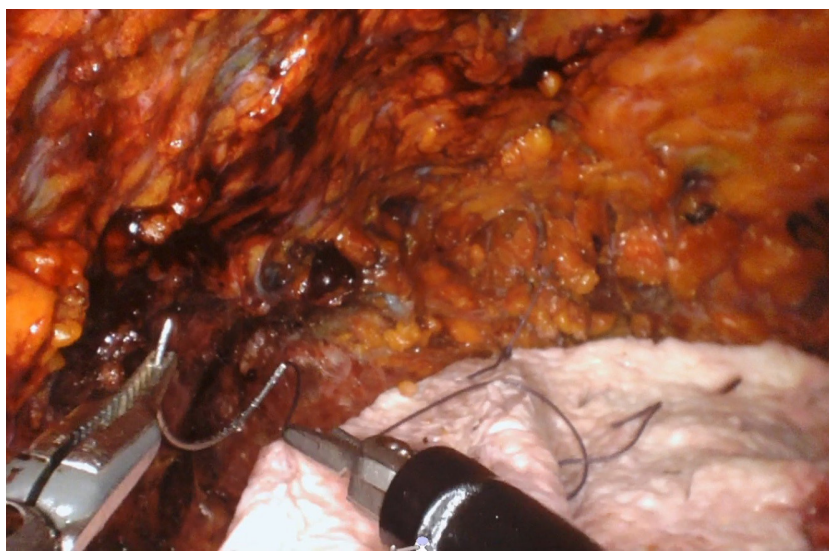

Fig. 4. Acellular dermal matrix sling fixation.

sibility of rippling and muscle action were also considered in this decision. After the same mastectomy procedure mentioned above, the pectoralis major muscle was elevated under direct vision, from the lateral border to the nipple level with caution. Further dissection to the inferomedial origin of the pectoralis major muscle had to be performed using the robotic system due to the interference of the chest wall curvature. During this procedure, a 6-mm Maryland Bipolar Forceps EndoWrist instrument (Intuitive Surgical Inc.) and a 6-mm Monopolar Permanent Cautery Spatula EndoWrist instrument were used for traction/counter-traction and dissection, respectively. Then with the right robotic arm, the ADM was sutured to the inframammary fold to create an ADM sling with Vicryl interrupted sutures using a Mega Suture Cut Needle Driver (Intuitive Surgical Inc.) (Fig. 4). After preparing the subpectoral pocket, two 200$\mathrm{mL}$ negative drainage systems were inserted, and irrigation with saline containing antibiotics was done. An expander was inserted into the subpectoral pocket, as the $\mathrm{ADM}$ sling serving as a

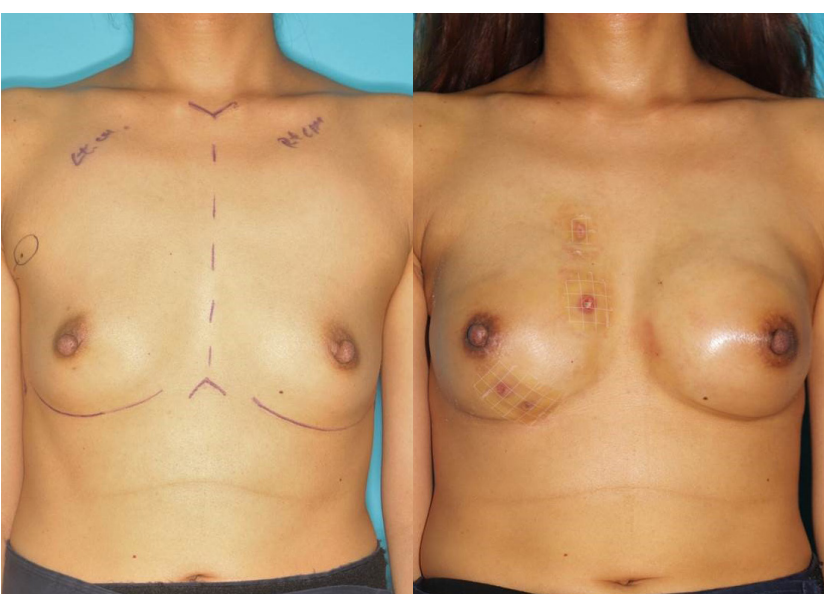

Fig. 5. Preoperative and postoperative frontal views (case 1). Preoperative and at 1 month postoperative.

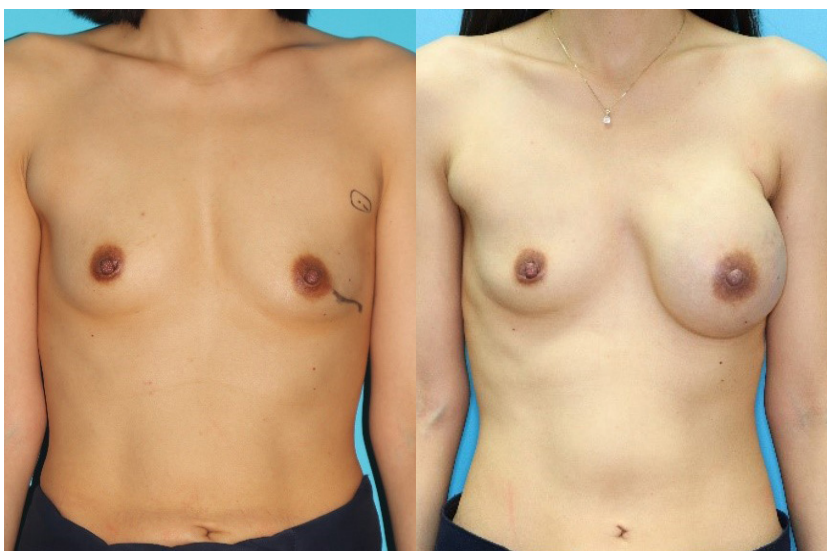

Fig. 6. Preoperative and postoperative frontal views (case 2). Preoperative and at 1 month postoperative, with $460 \mathrm{cc}$ inflated tissue expander.

supportive structure inferiorly. Intraoperatively, the expander was inflated with saline, considering skin tension. After suturing the $\mathrm{ADM}$ to the muscle, the operation was finished.

No conversion to open surgery, hematoma, total nipple or skin necrosis occurred. The first patient (case 1) experienced mild infection on a single breast, which resolved with intravenous antibiotic treatment without the need for implant removal. The second patient (case 2) did not experience any major/minor complications. In case of the operation time for breast reconstruction, the bilateral direct-to-implant case took about 4 hours while the expander insertion case took about 2 hours.

The second patient (case 2) has not undergone second-stage reconstruction at the time of publication. Preoperative and postoperative clinical photographs of the patients are shown in Figs. 5 and 6 . Both patients were satisfied with their results, especially with the unnoticeable scars (Fig. 7). 


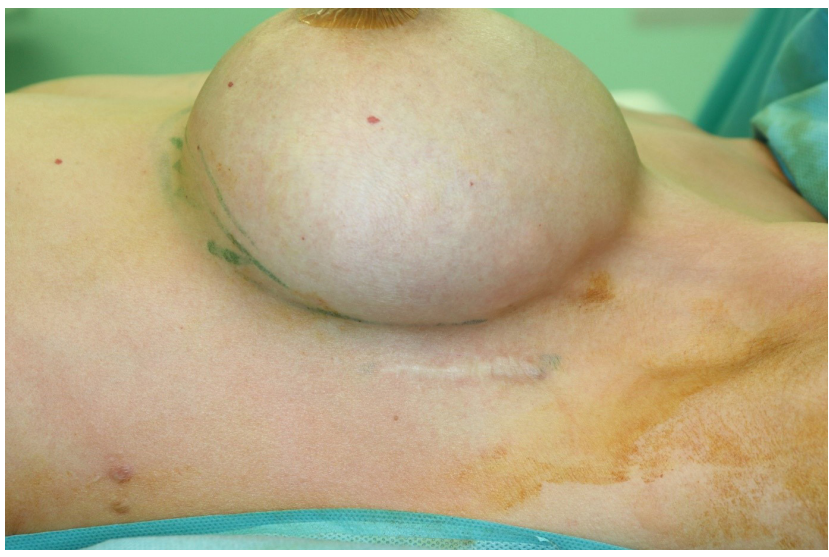

Fig. 7. Postoperative scar after 1 year.

\section{DISCUSSION}

Compared to other cancer-diagnosed patients, patients with early breast cancer are more interested in postoperative cosmetic results. This seems obvious regarding the improved diseasefree period and overall survival rates. With regard to aesthetic outcomes, R-NSM with IBR could be a new surgical method that ensures oncologic safety. Previous studies have shown that this method could be safe and feasible $[4,8]$.

Among the several advantages of the robotic approach are subtle manipulation, seven degrees of freedom of motion (including in/out, rotation, pitch/yaw at wrist, pitch/yaw at fulcrum, and grip strength), and being comparatively simple to learn [9]. However, there are some technical difficulties regarding the inadequate working space with bulky robot instruments.

The surgeon could experience familiarity using the new SP platform since it shares multiple features with prior multiport da Vinci platforms ( $\mathrm{S}, \mathrm{Si}$, and $\mathrm{Xi}$ models). It uses the same operative interface, including EndoWrist manipulators, high-definition three-dimensional visualization with magnification and scaled movement, and tremor reduction. Moreover, the incision length of mastectomy using da Vinci SP can be reduced to 2.5 $\mathrm{cm}$. However, to introduce an ADM-wrapped implant such as in our first case, we think at least $4 \mathrm{~cm}$ of incision is inevitable for use of around $300 \mathrm{cc}$ implant. In our institution we use $16 \times 16 \mathrm{~cm}$ sized ADM for wrapping, and it is impossible to fully wrap big sized prosthesis. In such cases when bigger implants must be used, it is important not only to make sure that the $\mathrm{ADM}$ does not get denuded during the insertion process but also to consider longer incisions if needed.

Of course, several technical advances have been made which enable the use of multiple robotic instruments through a single incision [10]. In these two cases, the smaller diameter of the da Vinci SP robot arms $(6 \mathrm{~mm})$ compared to other robotic systems
$(8 \mathrm{~mm})$ made procedures such as pectoralis major muscle elevation and $\mathrm{ADM}$ suture easier and more convenient. Especially, the da Vinci SP platform's novel modifications from previous models such as flexible camera, articulating instruments, and navigator guidance for real-time monitoring of instrument position allowed continuous adjustment and readjustment of the visual perspective. In the author's opinion, this surgical platform is the most appropriate device for carrying out mastectomy and breast reconstruction through a small sized single incision.

Prepectoral breast reconstruction is a modern concept that was reported to have not only promising cosmetic results but also decreased muscular dissection and related injuries [11]. This case has shown that both subpectoral/prepectoral breast reconstruction using the da Vinci SP Surgical Platform could be safe and feasible. To our knowledge, this study is the first to describe a clinical series using or not using the da Vinci SP robotic platform to perform da Vinci SP assisted NSM with IBR.

\section{NOTES}

\section{Conflict of interest}

No potential conflict of interest relevant to this article was reported.

\section{Ethical approval}

The study was approved by the Institutional Review Board of Severance Hospital (IRB No. 1-2019-0087) and performed in accordance with the principles of the Declaration of Helsinki. Institutional Review Board of Severance Hospital waived informed consent and approved the design of this retrospective study.

\section{Patient consent}

The patients provided written informed consent for the publication and the use of their images.

\section{Author contribution}

Conceptualization: SY Song, TS Roh. Methodology: OY Joo, HS Park, TS Roh. Project administration: SY Song. Visualization: OY Joo. Writing - original draft: OY Joo. Writing - review \& editing: OY Joo, SY Song.

\section{ORCID}

Oh Young Joo https://orcid.org/0000-0001-5736-4671

Hyung Seok Park https://orcid.org/0000-0001-5322-6036

Seoung Yong Song https://orcid.org/0000-0002-3145-7463

Tai Suk Roh 


\section{REFERENCES}

1. Selber JC, Baumann DP, Holsinger FC. Robotic latissimus dorsi muscle harvest: a case series. Plast Reconstr Surg 2012;129:1305-12.

2. Abramovici L, Cartier C, Pierre G, et al. Robot-assisted transaxillary thyroidectomy: surgical technique. Eur Ann Otorhinolaryngol Head Neck Dis 2015;132:153-6.

3. Toesca A, Peradze N, Manconi A, et al. Robotic nipple-sparing mastectomy for the treatment of breast cancer: feasibility and safety study. Breast 2017;31:51-6.

4. Toesca A, Peradze N, Galimberti V, et al. Robotic nipplesparing mastectomy and immediate breast reconstruction with implant: first report of surgical technique. Ann Surg 2017;266:e28-30.

5. Sarfati B, Honart JF, Leymarie N, et al. Robotic da Vinci Xiassisted nipple-sparing mastectomy: first clinical report. Breast J 2018;24:373-6.

6. Bertolo R, Garisto J, Gettman M, et al. Novel system for ro- botic single-port surgery: feasibility and state of the art in urology. Eur Urol Focus 2018;4:669-73.

7. Orosco RK, Tam K, Nakayama M, et al. Transoral supraglottic laryngectomy using a next-generation single-port robotic surgical system. Head Neck 2019;41:2143-7.

8. Park HS, Lee J, Lee DW, et al. Robot-assisted nipple-sparing mastectomy with immediate breast reconstruction: an initial experience. Sci Rep 2019;9:15669.

9. Ahn SJ, Song SY, Park HS, et al. Early experiences with robot-assisted prosthetic breast reconstruction. Arch Plast Surg 2019;46:79-83.

10. Dobbs RW, Halgrimson WR, Talamini S, et al. Single-port robotic surgery: the next generation of minimally invasive urology. World J Urol 2020;38:897-905.

11. Sigalove S, Maxwell GP, Sigalove NM, et al. Prepectoral implant-based breast reconstruction: rationale, indications, and preliminary results. Plast Reconstr Surg 2017;139:28794. 